\title{
Impacts of Multipath Fading on the Timing Synchronization of IEEE 802.11a Wireless LANs
}

\author{
Kun-Wah Yip, Tung-Sang Ng and Yik-Chung Wu \\ Department of Electrical and Electronic Engineering \\ The University of Hong Kong \\ Pokfulam Road, Hong Kong
}

\begin{abstract}
The timing-synchronization performance of IEEE 802.11a wireless LANs on multipath Rician fading channels is investigated. Simulation results yield the following observations. A higher Rician factor gives a better performance. At low signal-to-noise conditions, the multipath-diversity gain enables an improvement in the synchronization performance but at high signal-to-noise conditions, irreducible probabilities of synchronization failure, which cannot be reduced even if the signal power is increased, occur. A more dispersive channel gives a poorer synchronization performance, and the performance is poor for Rayleigh-fading environments.
\end{abstract}

\section{INTRODUCTION}

IEEE 802.11a wireless LANs [1] support packetized data transmission at a high rate up to 54Mbps. Before the data can be demodulated, the receiver needs to time-synchronize at the received data packet. Synchronization is accomplished by using the correlation technique to detect the time location of a known pilot symbol in the data packet.

Wireless LANs are usually operated inside buildings and it is known that indoor radio channels are characterized by the presence of multipath fading. In IEEE 802.11a wireless LANs, orthogonal frequency division multiplexing (OFDM) is used. The use of OFDM is advantageous because intersymbol interference (ISI) caused by the multipath spread can be removed in data demodulation by discarding the cyclic prefix, provided it is longer than the delay spread. A cyclic prefix of $800 \mathrm{~ns}$ is inserted into every data-carrying OFDM symbol so that a delay spread up to $800 \mathrm{~ns}$ can be tolerated. Since the delay spread of an indoor radio channel is typically around 200ns [2], this length of cyclic prefix is generally sufficient. On the other hand, the effect of multipath fading on the timing-synchronization performance of IEEE 802.11a wireless LANs is less well-understood. By analyzing the correlation property of the pilot OFDM symbol, it can be shown that multipath components with delays greater than $50 \mathrm{~ns}$ become resolvable during the correlation process of timing synchronization. The resolvable components are independently faded so that diversity combining is possible and the synchronization performance can be enhanced. However, ISI is also introduced, causing a negative impact on the synchronization performance. Notice that when the correct synchronization point cannot be established, data

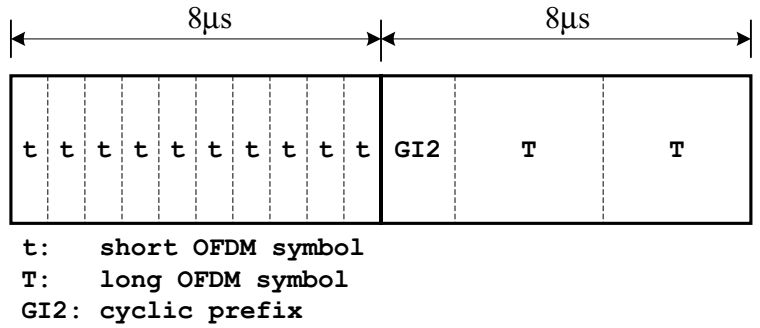

Fig. 1. Preamble structure of an IEEE 802.11a data packet.

carried in the OFDM symbols cannot be demodulated. The consequence of synchronization failure is the need to retransmit data packets even if the OFDM symbol structure is indeed able to tolerate the delay spread of the situation under consideration. It follows that the probability of synchronization failure is an important factor in the determination of the wireless-LAN performance.

Although there have been many works, such as [3]-[6], concerning synchronization aspects of OFDM systems, these works are specific to the proposed systems therein and cannot provide insights into the synchronization performance of IEEE 802.11a wireless LANs. In this paper, we investigate the timing-synchronization performance of IEEE 802.11a wireless LANs operating on multipath Rician fading channels. Measurement results have shown that multipath Rician fading is appropriate to model the characteristic of indoor radio channels [7]-[9]. The synchronization performance is investigated by means of simulation. The simulation model is given in Section II. Numerical results are presented in Section III. Section IV concludes the paper.

\section{TIMING-SYNCHRONIZATION MODEL}

A $16 \mu$ s preamble having a structure shown in Fig. 1 is inserted at the beginning of each data packet. The preamble is divided into two subpreambles. The first one consists of ten identical, short OFDM symbols each having a length of $800 \mathrm{~ns}$. The second one comprises two long OFDM symbols each of length $3.2 \mu$ s preceded by a $1.6 \mu$ s cyclic prefix. It is required to detect the end of the first subpreamble. This time reference enables the receiver to locate the time instant in the second subpreamble at which the FFT window for frequency- 
offset compensation begins, and also the starting point of the FFT time window for data demodulation in the rest of the packet. To detect the end of the first subpreamble, the receiver can correlate the received signal with the short OFDM symbol. The presence of a correlation peak indicates that the first subpreamble has not passed while the absence of an expected correlation peak is an indication that the current time position is at $800 \mathrm{~ns}$ in the second subpreamble. A sliding correlator is required and a sampling rate of $20 \mathrm{MHz}$, suggested [1], is used. Note that a large frequency offset may be present during correlation. It is required that the deviation of the transmitted-signal center frequency is within $\pm 20 \mathrm{ppm}$ [1]. If the receiver oscillator also has a frequency uncertainty of $\pm 20 \mathrm{ppm}$ and the operating frequency is $5 \mathrm{GHz}$. The frequency offset is within $\pm 200 \mathrm{kHz}$.

The OFDM signal specified in the standard [1] comprises 52 subcarriers numbered from -26 to +26 with no signal transmission at the zeroth subcarrier. The complex envelope of the preamble, $s(t)$, is given by [1]

$$
\begin{aligned}
s(t) & =\sqrt{2 P} \times\left\{\sum_{k=-26}^{26} B_{k} e^{j 2 \pi k \Delta_{f} t} w\left(0, T_{p 1}\right)\right. \\
& \left.+\sum_{k=-26}^{26} C_{k} e^{j 2 \pi k \Delta_{f}\left(t-T_{p 1}-T_{G I 2}\right)} w\left(T_{p 1}, T_{p 1}+T_{p 2}\right)\right\}
\end{aligned}
$$

where (a) $T_{p 1}=8 \mu \mathrm{s}$ and $T_{p 2}=8 \mu \mathrm{s}$ are the lengths of the first and second subpreambles, respectively, (b) $T_{G I 2}=1.6 \mu \mathrm{s}$ is the length of the cyclic prefix of the second subpreamble, (c) $w\left(t_{1}, t_{2}\right)$ is the raised-cosine window detailed in [1], (d) $P$ is the signal power, (e) $\Delta_{f}=312.5 \mathrm{kHz}$ is the subcarrier spacing, and (f) $B_{k}=52^{-1 / 2} S_{k}$ and $C_{k}=52^{-1 / 2} L_{k}$ are pilot symbols used to generate the short and long OFDM symbols, respectively, wherein $S_{k}$ and $L_{k}$ are detailed in [1]. We mention that $B_{0}=C_{0}=0$, and $B_{k}=0$ for $k$ not a multiple of 4. The signal is transmitted through a multipath Rician fading channel. Since the signal is bandlimited, it is known that the channel can be modeled by a tapped delay-line model $[10, \mathrm{pp} .795]$. The channel impulse response, $h(t)$, is given by

$$
h(t)=A e^{j \phi} \delta(t)+\sum_{\ell=0}^{L-1} \alpha_{\ell} e^{j \theta_{\ell}} \delta\left(t-\ell T_{B}\right)
$$

where (a) $A$ and $\phi$ are the deterministic gain and random phase, respectively, of the direct path, (b) $\alpha_{\ell}$ and $\theta_{\ell}$ are the gain and phase of the $\ell$ th faded path, modeled by, respectively, a Rayleigh-distributed random variable with a variance $\overline{\alpha_{\ell}^{2}}$ and a uniform random variable over $[0,2 \pi)$, (c) $L$ is the number of resolvable paths or the number of taps in the channel model, and (d) $T_{B}$ is the inverse of the signal bandwidth. We use $T_{B}=50 \mathrm{~ns}$ since the signal occupies approximately $20 \mathrm{MHz}[1]$. Note that $\alpha_{\ell}$ and $\theta_{\ell}, \ell=0,1$, $\ldots, L-1$, are statistically independent. The Rician factor,
$K$, is given by $K=A^{2} / \sum_{\ell=0}^{L-1} \overline{\alpha_{\ell}^{2}}$. The complex envelope of the received signal after RF filtering, $r(t)$, is given by

$$
r(t)=\eta(t)+e^{j 2 \pi f_{o f f} t}\{h(t) * s(t)\}
$$

where $*$ denotes convolution, $\eta(t)$ is the complex-Gaussian noise, and $f_{\text {off }}$ is the frequency offset. The noise power is computed by $\frac{1}{2} E\left\{|\eta(t)|^{2}\right\}=N_{0} W$ where $N_{0}$ is the one-sided noise power spectral density of additive white Gaussian noise and $W$ is the noise bandwidth of the RF filter. Since the signal energy is concentrated within a bandwidth of $18 \mathrm{MHz}$ [1], we consider that $W=18 \mathrm{MHz}$. The received signal is digitized at a sampling rate of $1 / T_{\text {sam }}$ samples per second with $T_{\text {sam }}^{-1}=20 \mathrm{MHz}$. The $n$th signal sample, $r_{n}$, is given by $r_{n}=$ $r\left(n T_{\text {sam }}+\tau_{\text {sam }}\right)$ where $\tau_{\text {sam }}$ is the fractional time offset modeled by a uniform random variable over $\left[-\frac{1}{2} T_{\text {sam }}\right.$, $\left.\frac{1}{2} T_{\text {sam }}\right]$. It is assumed that the correlations of noise components in $r_{n}$ 's are negligible.

A sliding correlator is used to process the received signal samples. We denote $T_{s s}=800 \mathrm{~ns}$ as the length of the short OFDM symbol. The correlator output obtained at time $n T_{\text {sam }}$ $+\tau_{\text {sam }}$ is computed by

$$
\Xi_{n}=\sum_{m=1}^{M} r_{n-M+m} g_{m}^{*}
$$

where $M=T_{s s} / T_{\text {sam }}=16$, and $g_{m}=\sum_{k=-26}^{26} B_{k} e^{j 2 \pi k \Delta_{f} m T_{s a m}}$. The synchronization procedure is as follows. The time location of the correlation peak and its $\left|\Xi_{n}\right|^{2}$ value are identified by searching for the maximum value of $\left|\Xi_{n}\right|^{2}$ over the obtained correlation results. Note that selecting the maximum $\left|\Xi_{n}\right|^{2}$ value as the correlation peak corresponds to utilizing multipath diversity with selection combining. After the correlation peak is located, the next $M$ th correlation result is compared with the maximum value of $\left|\Xi_{n}\right|^{2}$ to determine if another correlation peak is present. The search for expected correlation peaks continues until an absence is identified, in which the second subpreamble is entered. Note that a new correlation result is generated every sampling instant so that the maximum value of $\left|\Xi_{n}\right|^{2}$ and the corresponding time location are regularly updated. Let $\mu_{i}$ and $n_{i}$ be the maximum value of $\left|\Xi_{n}\right|^{2}$ and the corresponding time location, respectively, recorded at the $i$ th sampling instant but excluding the current correlation result. It follows that $\mu_{i}=\max _{n<i}\left|\Xi_{n}\right|^{2}$. The end of the first subpreamble is declared at the sampling instant $i-M$ if $n-n_{i}$ is a multiple of $M$ and

$$
\beta \mu_{i}>\left|\Xi_{i}\right|^{2}
$$

is satisfied, where $\beta \in(0,1)$ is a given threshold value. 
(a)

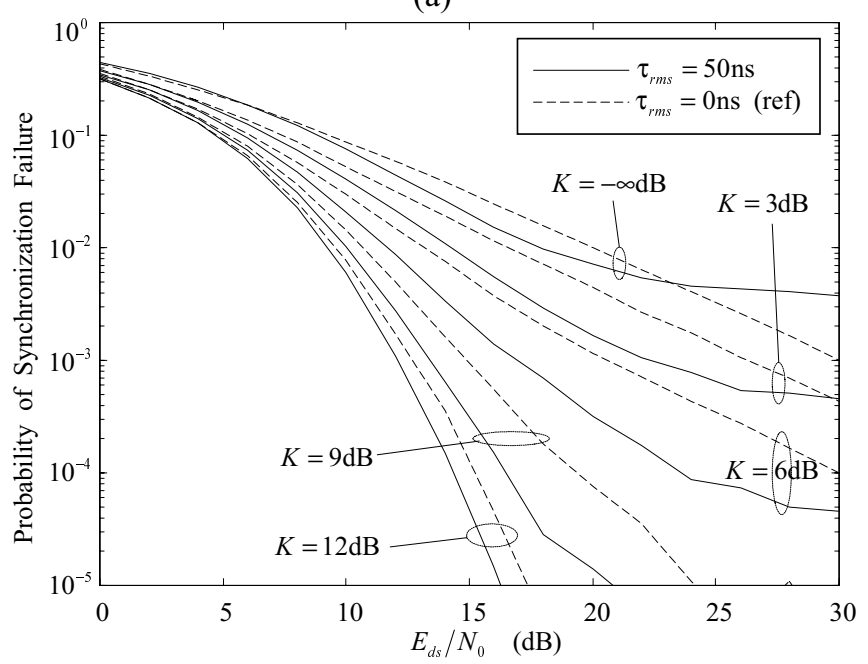

(c)

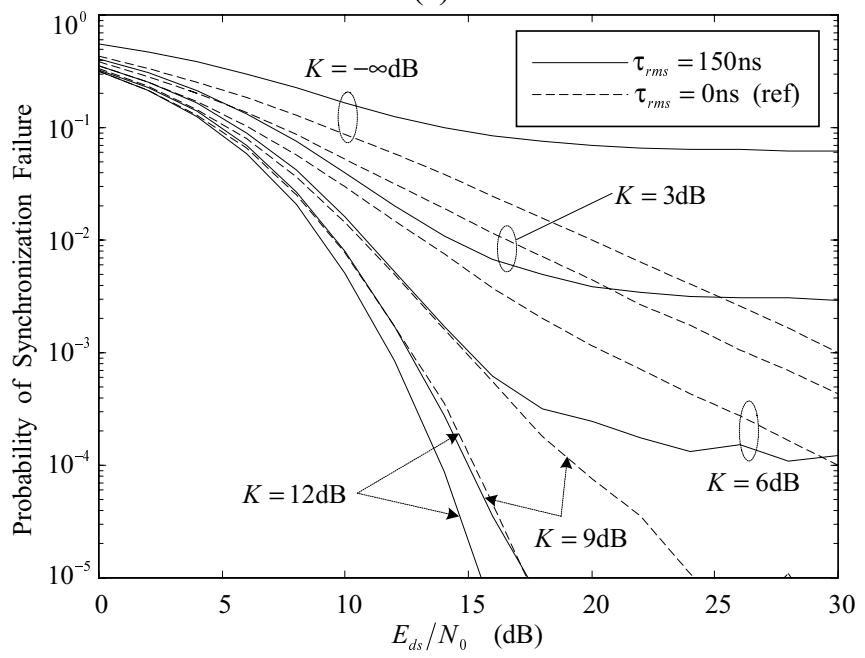

(b)

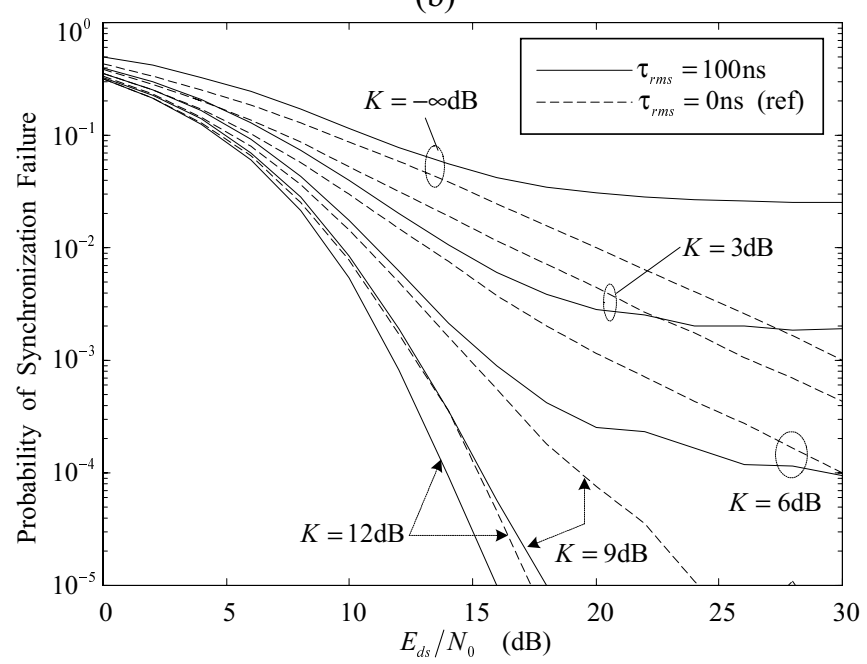

(d)

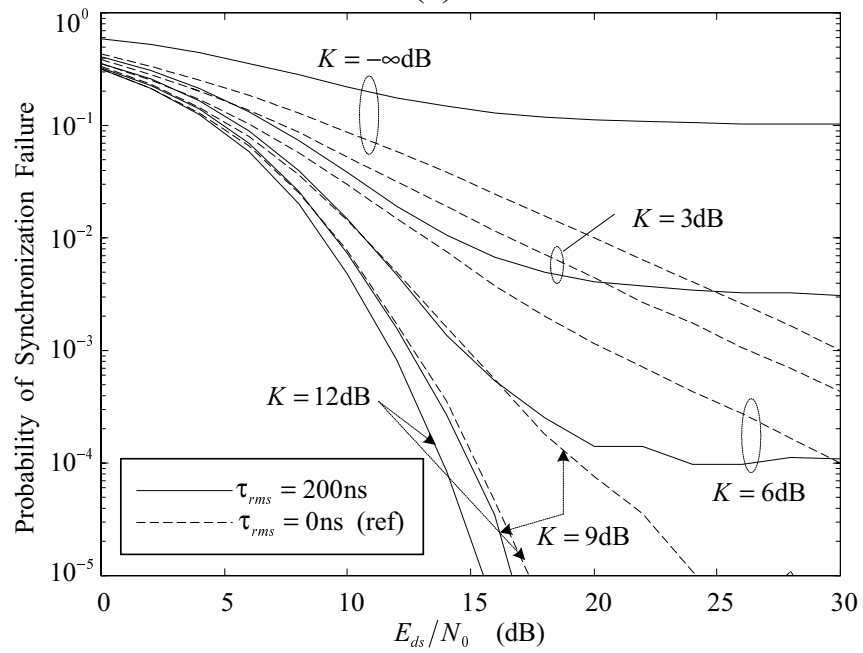

Fig. 2. $P_{\text {fail }}$ against $E_{d s} / N_{0}$ for $\tau_{r m s}=$ (a) $50 \mathrm{~ns}$, (b) $100 \mathrm{~ns}$, (c) $150 \mathrm{~ns}$, and (d) $200 \mathrm{~ns}$. The curves for $\tau_{r m s}=0 \mathrm{~ns}$ are plotted for reference.

\section{SimULATION RESUlTS AND DISCUSSION}

We follow the suggestion of the IEEE 802.11a specifications [1] that the last three short OFDM symbols are used for timing synchronization. It follows that correlation peaks occur at $n=8 M, 9 M$ and $10 M$, or within a small region. Since OFDM is rather robust to small time offsets as a result of the inclusion of cyclic prefix (800ns) in the data format [11], in simulation this small region was set to be $300 \mathrm{~ns}$ in length. At $n=11 M$ or its vicinity, the value of $\left|\Xi_{n}\right|^{2}$ drops significantly. An event of false alarm arises when (5) is satisfied for $n=9 M$ or $10 M$ or their vicinities. A miss occurs when (5) is not satisfied for $n=11 M$ or around. A synchronization failure is an event where either a false alarm or a miss occurs. In this work, we consider only the cases that $\beta=0.25,0.5$ and 0.75 since these choices of $\beta$ are appropriate for digital implementation of synchronization circuits.

The channel dispersion is modeled by an exponential function. It is assumed that the channel has a unity gain so that $A^{2}+\sum_{\ell=0}^{L-1} \overline{\alpha_{\ell}^{2}}=1$. We calculate $A$ and $\overline{\alpha_{\ell}^{2}}$ by

$$
A=\sqrt{\frac{K}{K+1}}
$$


and

$$
\overline{\alpha_{\ell}^{2}}=\frac{1}{K+1} \int_{\ell T_{B}}^{(\ell+1) T_{B}} \tau_{r m s}^{-1} e^{-\tau / \tau_{r m s}} d \tau
$$

where $\tau_{r m s}$ is the RMS delay spread of the Rayleigh-faded components. In the simulation, we considered that $\tau_{r m s} \leq$ 200ns, and set $L=10$. We consider that $K$ ranges from $-\infty \mathrm{dB}$ to $12 \mathrm{~dB}$ [7]-[9]. The probability of synchronization failure, $P_{\text {fail }}$, was simulated against $E_{d s} / N_{0}$ where $E_{d s}$ is the energy of a data symbol. The signal-to-noise ratio (SNR), given by $\mathrm{SNR}=P /\left(N_{0} W\right)$, is related to $E_{d s} / N_{0}$ by $\mathrm{SNR}=$ $52 /\left(T_{d s} W\right) \times E_{d s} / N_{0}$ where $T_{d s}=3.2 \mu \mathrm{s}$ is the duration of a data symbol excluding the cyclic prefix. We consider the cases that $P_{\text {fail }}=10^{-3}$ and $P_{\text {fail }}=10^{-4}$. Each $P_{\text {fail }}$ value was generated by performing $10^{6}$ simulation runs.

Table I lists the simulated $P_{\text {fail }}$ values for $f_{\text {off }} \in\{-$ $210 \mathrm{kHz},-90 \mathrm{kHz}, 0 \mathrm{kHz}, 90 \mathrm{kHz}, 210 \mathrm{kHz}\}$ and $\beta \in\{0.25$, $0.5,0.75\}$ at $E_{d s} / N_{0}=20 \mathrm{~dB}, K=6 \mathrm{~dB}$ and $\tau_{r m s} \in\{50 \mathrm{~ns}$, $100 \mathrm{~ns}\}$. It is apparent that the choice of $\beta=0.5$ yields the lowest $P_{\text {fail }}$ among the three cases of $\beta$ under consideration. In the rest of the paper, we consider $\beta=0.5$. Results of Table I also show that, for $\beta=0.5$, the difference of $P_{\text {fail }}$ for different $f_{\text {off }}$ at a given $\beta$ is within a factor of three and the variation of $P_{\text {fail }}$ is in general not significant. Therefore, in the investigation that follows, we consider representative values of $P_{\text {fail }}$ computed by setting $f_{\text {off }}=0 \mathrm{kHz}$.

Figs. 2(a)-2(d) plot the simulated $P_{\text {fail }}$ values against $E_{d s} / N_{0}$ for $\tau_{r m s}=50 \mathrm{~ns}, 100 \mathrm{~ns} 150 \mathrm{~ns}$ and $200 \mathrm{~ns}$, respectively, under different conditions of $K$. In each figure, $P_{\text {fail }}$ for $\tau_{r m s}=0 \mathrm{~ns}$ is also plotted for reference. The following observations are made.

1. As expected, the synchronization performance is better in the presence of a higher Rician factor.

2. At low $E_{d s} / N_{0}$ conditions $(<20 \mathrm{~dB})$ and $K \in\{3 \mathrm{~dB}, 6 \mathrm{~dB}$, $9 \mathrm{~dB}, 12 \mathrm{~dB}\}$, the $P_{\text {fail }}$ values for multipath fading channels (with nonzero $\tau_{r m s}$ ) are lower than those for frequency-nonselective fading channels $\left(\tau_{r m s}=0 \mathrm{~ns}\right)$. The performance improvement is due to the multipath diversity. At $K=-\infty \mathrm{dB}$, the presence of multipath diversity yields a better performance only for the case that $\tau_{\text {rms }}=50 \mathrm{~ns}$.

3. At high $E_{d s} / N_{0}$ conditions, the values of $P_{\text {fail }}$ cannot be reduced even if the signal power is increased. It is due to the presence of channel dispersion, which results in ISI and leads to the occurrence of irreducible probabilities of synchronization failure. The irreducible $P_{\text {fail }}$ limits the best synchronization performance that can be achieved for IEEE 802.11a wireless LANs.
TABLE I

$P_{\text {fail }}$ FOR DifFerent Combinations of $f_{\text {off }}$ AND $\beta$ AT $E_{d s} / N_{0}=20 \mathrm{DB}$

\begin{tabular}{rccc}
\multicolumn{3}{c}{ AND $K=6 \mathrm{DB}}$. \\
\hline $\begin{array}{c}f_{\text {off }} \\
(\mathrm{kHz})\end{array}$ & $\beta$ & $P_{\text {fail }}$ for & $P_{\text {fail }}$ for \\
\hline-210 & 0.25 & $1.47 \times 10^{-3}$ & $3.60 \times 10^{-3}$ \\
-90 & 0.25 & $2.11 \times 10^{-3}$ & $4.78 \times 10^{-3}$ \\
0 & 0.25 & $1.53 \times 10^{-3}$ & $2.66 \times 10^{-3}$ \\
90 & 0.25 & $1.38 \times 10^{-3}$ & $2.04 \times 10^{-3}$ \\
210 & 0.25 & $2.31 \times 10^{-3}$ & $3.08 \times 10^{-3}$ \\
\hline-210 & 0.50 & $4.60 \times 10^{-5}$ & $2.02 \times 10^{-4}$ \\
-90 & 0.50 & $4.10 \times 10^{-5}$ & $1.90 \times 10^{-4}$ \\
0 & 0.50 & $3.90 \times 10^{-5}$ & $1.01 \times 10^{-4}$ \\
90 & 0.50 & $9.10 \times 10^{-5}$ & $2.29 \times 10^{-4}$ \\
210 & 0.50 & $7.40 \times 10^{-5}$ & $1.89 \times 10^{-4}$ \\
\hline-210 & 0.75 & $1.23 \times 10^{-3}$ & $6.16 \times 10^{-3}$ \\
-90 & 0.75 & $0.07 \times 10^{-3}$ & $1.13 \times 10^{-3}$ \\
0 & 0.75 & $1.27 \times 10^{-3}$ & $4.29 \times 10^{-3}$ \\
90 & 0.75 & $2.13 \times 10^{-3}$ & $4.11 \times 10^{-3}$ \\
210 & 0.75 & $1.71 \times 10^{-3}$ & $5.00 \times 10^{-3}$ \\
\hline
\end{tabular}

TABLE II

Multipath-diversity gains at $P_{\text {fail }}=10^{-3}$ and $P_{\text {fail }}=10^{-4}$.

\begin{tabular}{|c|c|c|c|}
\hline \multirow[b]{2}{*}{$\tau_{r m s}(\mathrm{~ns})$} & \multirow[b]{2}{*}{$K(\mathrm{~dB})$} & \multicolumn{2}{|c|}{ Diversity gain $(\mathrm{dB})$ at } \\
\hline & & $P_{\text {fail }}=10^{-3}$ & $P_{\text {fail }}=10^{-4}$ \\
\hline \multirow{5}{*}{50} & $-\infty$ & NR & NR \\
\hline & 3 & 3.9 & NR \\
\hline & 6 & 3.6 & 6.3 \\
\hline & 9 & 1.5 & 2.7 \\
\hline & 12 & 0.9 & 0.9 \\
\hline \multirow{5}{*}{100} & $-\infty$ & NR & NR \\
\hline & 3 & NR & NR \\
\hline & 6 & 4.9 & $*$ \\
\hline & 9 & 2.0 & 3.8 \\
\hline & 12 & 1.2 & 1.6 \\
\hline \multirow{5}{*}{150} & $-\infty$ & NR & NR \\
\hline & 3 & NR & NR \\
\hline & 6 & 5.4 & $*$ \\
\hline & 9 & 2.2 & 4.0 \\
\hline & 12 & 1.2 & 1.6 \\
\hline \multirow{5}{*}{200} & $-\infty$ & NR & NR \\
\hline & 3 & NR & NR \\
\hline & 6 & 5.8 & $*$ \\
\hline & 9 & 2.3 & 4.1 \\
\hline & 12 & 1.2 & 1.6 \\
\hline
\end{tabular}

NR not realizable

* estimate not reliable due to ringing of simulated data

4. By examining the irreducible $P_{\text {fail }}$ values under different 
conditions of $\tau_{r m s}$, it is apparent that a more dispersive channel yields a poorer synchronization performance. This result indicates that the performance improvement due to a higher degree of multipath diversity cannot compensate for the degradation due to more ISI.

5. It is apparent that the synchronization performance is poor in Rayleigh-fading environments $(K=-\infty \mathrm{dB})$. In all cases of nonzero $\tau_{r m s}$, the irreducible $P_{\text {fail }}$ values cannot even be reduced to $10^{-3}$. The poor synchronization performance reduces the capabilities of IEEE 802.11a wireless LANs in applications using hand-held portable computing devices.

Table II provides a summary on the multipath-diversity gain, which is expressed in terms of the reduction of $E_{d s} / N_{0}$ to achieve a $P_{\text {fail }}$ of $10^{-3}$ or $10^{-4}$. The results show that it is not possible to realize a multipath-diversity gain in most cases of $\tau_{r m s}$ when $K=-\infty \mathrm{dB}$ and $3 \mathrm{~dB}$. It is because the irreducible $P_{\text {fail }}$ values are greater than $10^{-3}$. For $K=6 \mathrm{~dB}$, a quite sizable multipath-diversity gain ranging from around $3.6 \mathrm{~dB}$ to $6.3 \mathrm{~dB}$ can be obtained. Beyond $K=6 \mathrm{~dB}$, the multipathdiversity gain decreases as $K$ increases.

\section{CONCLUSIONS}

Extensive simulation has been conducted for investigating the timing-synchronization performance of IEEE 802.11a wireless LANs on frequency-selective Rician fading channels. In the investigation, $\beta=0.5$ has been selected. It has been found that the impact of frequency offset is in general not significant. Simulation results lead to the following observations. A higher Rician factor yields a better synchronization performance. At low $E_{d s} / N_{0}$ conditions, the multipath-diversity gain enables an improvement of the synchronization performance. However, irreducible probabilities of synchronization failure occur at high $E_{d s} / N_{0}$ ratios. It has also been shown that a more dispersive channel results in a poorer synchronization performance. In addition, the synchronization performance is poor in a Rayleigh-fading environment.

\section{REFERENCES}

[1] IEEE Computer Society, IEEE Std 802.11a-1999, 30 Dec. 1999.

[2] A. A. M. Saleh and R. A. Valenzuela, "A statistical model for indoor multipath propagation," IEEE J. Select. Areas Commun., vol. 5, pp. 128-137, Feb. 1987.

[3] L. Házy and M. El-Tanany, "Synchronization of OFDM systems over frequency selective fading channels," Proc. IEEE VTC'97, pp. 2094-2098, May 4-7, 1997.

[4] R. J. Castle, "A two-stage synchronization technique for OFDM," Proc. IEEE VTC'99 - Fall, pp. 683-687, Sep. 19-22, 1999.

[5] T. Wakutsu and M. Serizawa, "A carrier frequency offset and timing offset detection scheme for OFDM systems utilizing pilot sub-carriers," IEICE Trans. Commun., vol. E83-B, pp. 1854-1863, Aug. 2000.

[6] F. Tufvesson, M. Faulkner, P. Hoeher and O. Edfors, "OFDM time and frequency synchronization by spread spectrum pilot technique," Proc. IEEE Commun. Theory Mini-Conf., pp. 115119, Jun. 6-10, 1999.

[7] R. J. C. Bultitude, "Measurement, characterization and modeling of indoor $800 / 900 \mathrm{MHz}$ radio channels for digital communications," IEEE Commun. Mag., vol. 25, no. 6, pp. 512, Jun. 1987.

[8] T. S. Rappaport and C. D. McGillem, "UHF fading in factories," IEEE J. Select. Areas Commun., vol. 7, pp. 40-48, Jan. 1989.

[9] S.-C. Kim, H. L. Bertoni and M. Stern, "Pulse propagation characteristics at $2.4 \mathrm{GHz}$ inside buildings," IEEE Trans. Veh. Technol., vol. 45, pp. 579-592, Aug. 1996.

[10] J. G. Proakis, Digital Communications, 3rd ed., New York: McGraw-Hill, 1995.

[11] R. van Nee and R. Prasad, OFDM for Wireless Multimedia Communications, ch. 4.4, Boston: Artech-House, 2000. 